\title{
Sobrevida postoperatoria en pacientes nonagenarios. Estudio pronóstico en cohorte retrospectiva 2001-2010, Clínica Dávila
}

\author{
DAGOBERTO OJEDA, FLORENCE GAZABATT, PATRICIA CISTERNAS, \\ FRANCISCA FOLCH $^{\text {a }}$, CHRISTOPHER DEMPSTER ${ }^{\mathrm{a}}$
}

Servicio de Anestesiología,
Clínica Dávila, Santiago
de Chile.
anterno(a) de Medicina,
Facultad de Medicina
Universidad de los Andes.
Santiago, Chile.

Trabajo financiado por el Servicio de Anestesiología de Clínica Dávila.

Los autores declaran no tener conflictos de intereses en este manuscrito.

Recibido el 12 de octubre de 2011, aceptado el 16 de agosto de 2012 .

Correspondencia a:

Dr. Dagoberto Ojeda. Servicio de Anestesiología, Clínica Dávila, Santiago Chile. E-mail: eojedadinamarca@ gmail.com

\section{Postoperative survival among nonagenarians. A retrospective study}

Background: As the Chilean population ages, anesthesiologists are regularly faced with elderly and even nonagenarian people undergoing surgical procedures. Aim: To determine the postoperative survival time in nonagenarians and its risk factors at a private clinic. Material and Methods: Review of medical records of the clinic, searching for patients aged 90 years or older, which were subjected to a surgical procedure between 2001 and 2012. Certification of survival or death was obtained from the clinical records or death certification at the National Identification Service. Survival analysis was made using Kaplan-Meier and Gompertz regression. Results: The medical records of 167 patients, aged 90 to 101 years (64\% women), were reviewed. Sixty four percent had an underlying cardiovascular disease; in 37\%, cognitive impairment. Hip fracture surgery was the most common procedure. One intraoperative death occurred. Five percent of patients died one month after surgery. The median survival time was two years and the longest, seven years. According to Gompertz probability regression, the predictors of death were the presence of cardiac disease (Hazard ratio (HR): 1.91, 95\% confidence intervals (95\% CI): 1.16; 3.16), cognitive impairment (HR: 2.10, 95\% CI: 1.32; 3,22), cancer (HR:2.10, 95\% CI: 1.32; 3.22), requirement of transfusion (HR: 1.79, 95\% CI: 1.13; 2.83) and an American Society of Anesthesiologists (ASA) Class III classification (HR: 1.95, IC95\%: 1.21; 3.15). Conclusions: In nonagenarian patients undergoing surgery; $50 \%$ mortality was observed 2 years after surgery. The presence of cardiac disease, cognitive impairment, cancer, transfusion and a Class III ASA classification were predictors of death.

(Rev Med Chile 2013; 141: 34-40).

Key words: Aged, 80 an over; Prognosis; Surgery.
L a llamada "transición epidemiológica" ha producido un cambio en la estructura por edad de la población chilena, aumentando la proporción de adultos mayores. El censo del año 2002 demostró que los mayores de 90 años aumentaron de 25.682 a 38.556 en relación al censo previo, conformando el $0,26 \%$ del total de la población del país ${ }^{1}$. Esto, sumado al desarrollo y avances en medicina, ha causado que los anestesió- logos deban enfrentarse cada vez más a pacientes ancianos ${ }^{2}$. El aumento del número de nonagenarios sometidos a cirugía también se explica por la disminución de la mortalidad postoperatoria en este grupo de pacientes ${ }^{2}$, Djokovic ${ }^{3}$ reportó una disminución de la mortalidad perioperatoria en pacientes de 80 años y más, desde $20 \%$ a $10 \%$ entre las décadas de 1960-69 y 1970-79. Este grupo de pacientes significa desde el punto de vista 
Sobrevida postoperatoria en nonagenarios - D. Ojeda et al

económico un importante aumento del gasto en salud $^{2}$ por tratarse de una población afectada de patologías degenerativas severas y crónicas. En Chile no existen reportes acerca de la mortalidad y pronóstico en pacientes de 90 años de edad y más; por ello se decidió realizar un estudio con el propósito de investigar la sobrevida postoperatoria de los nonagenarios y los factores pronósticos de ésta.

\section{Material y Método}

El diseño epidemiológico correspondió a un estudio de cohorte histórica. Se buscó en los registros médicos a los pacientes de 90 años de edad o más, que se hubieran operado entre el $1^{\circ}$ de enero de 2001 y el 31 de agosto de 2010 (fecha de término del estudio) en la Clínica Dávila, en Santiago de Chile. Se revisaron las fichas clínicas y se registraron las siguientes variables: edad, sexo, diagnóstico, tipo de cirugía y anestesia, estado físico según la clasificación de la American Society of Anesthesiologists $(A S A)^{4}$, cirugía de urgencia o electiva, presencia de patología agregada cardiaca, diabetes, hipertensión arterial, renal, hematológica, neurológica, estadía en una unidad de cuidado intensivo, cáncer, días de hospitalización. El tiempo de sobrevida fue consignado en días desde la primera operación (en el caso de que hubiera más de una) y se consideró vivo a todo paciente que se hubiera operado en el período señalado y que estuviera vivo al 31 de agosto de 2010 y muerto a todo paciente que se hubiera operado en dicho período y hubiera fallecido antes del 31 de agosto de 2010. La fecha de muerte se rescató de la ficha clínica o del certificado de defunción emitido por el registro civil. Se estimaron curvas de sobrevida por el método de Kaplan-Meier ${ }^{5}$ y, dado que el tiempo de sobrevida se amoldó a una distribución de probabilidad de Gompertz ${ }^{5}$, se utilizó un modelo de riesgos proporcionales paramétrico basado en esta distribución para evaluar los posibles factores pronósticos de muerte a todo tiempo, tanto individualmente como en forma multivariante. La evaluación final multivariante de los factores pronósticos de muerte se logró mediante un proceso step-wise con probabilidad de retención de $0,05 \%$. La variación en las tasas de anestesia en pacientes nonagenarios en el tiempo se realizó a través de regresión de Poisson. El análisis estadístico se realizó en Stata 10. El nivel para considerar un resultado estadísticamente significativo fue de $5 \%$.

\section{Resultados}

Ciento sesenta y siete pacientes nonagenarios fueron operados durante el período comprendido entre los años 2001 y 2010. La cohorte estudiada se caracterizó por un predominio del sexo femenino, pertenecer a las clases I y II de la clasificación de ASA, (o sea eran sanos o portadores de patologías crónicas estables), el uso de anestesia regional y de cirugía de urgencia (Tabla 1).

Estos pacientes nonagenarios tenían una alta prevalencia $(64 \%)$ de patología cardiaca (insuficiencia cardiaca, cardiopatía coronaria, arritmias), hipertensión arterial (63\%) y trastornos neurológicos como Alzheimer, demencia senil y deterioro psico-orgánico cerebral (37\%). La patología asociada menos prevalente fue la diabetes mellitus (12\%) (Figura 1).

El procedimiento quirúrgico más frecuente en esta cohorte fue la corrección de una fractura de cadera (Figura 2).

Hubo una muerte intraoperatoria, en relación a la cementación de una prótesis de cadera. La mortalidad a los 30 días del postoperatorio fue de $5 \%$. La mediana del tiempo de sobrevida de la cohorte fue de 2 años; vale decir, el 50\% de los pacientes sobrevivió a lo más 2 años y $75 \%$ de los pacientes fallecieron a los 5 años de seguimiento. El tiempo de sobrevida postoperatorio máximo fue de 7 años y 7 meses. La Figura 3 muestra la curva de sobrevida postoperatoria de toda la cohorte de

\section{Tabla 1. Características clínicas de nonagenarios} quirúrgicos

\begin{tabular}{|lc|}
\hline Característica & Resultado \\
\hline Edad en años (Mediana; min.-máx) & $92 ; 90-101$ \\
Sexo femenino (\%) & 64 \\
\hline ASA* (\%) & \\
I & 8 \\
II & 53 \\
III & 33,5 \\
IV & 4,8 \\
V & 0,7 \\
Tipo de anestesia (\%) & \\
Local y/o sedación & 27 \\
Regional & 50 \\
General & 23 \\
Cirugía de Urgencia (\%) & 60 \\
\hline
\end{tabular}

${ }^{*}$ American Society of Anesthesiologists. 


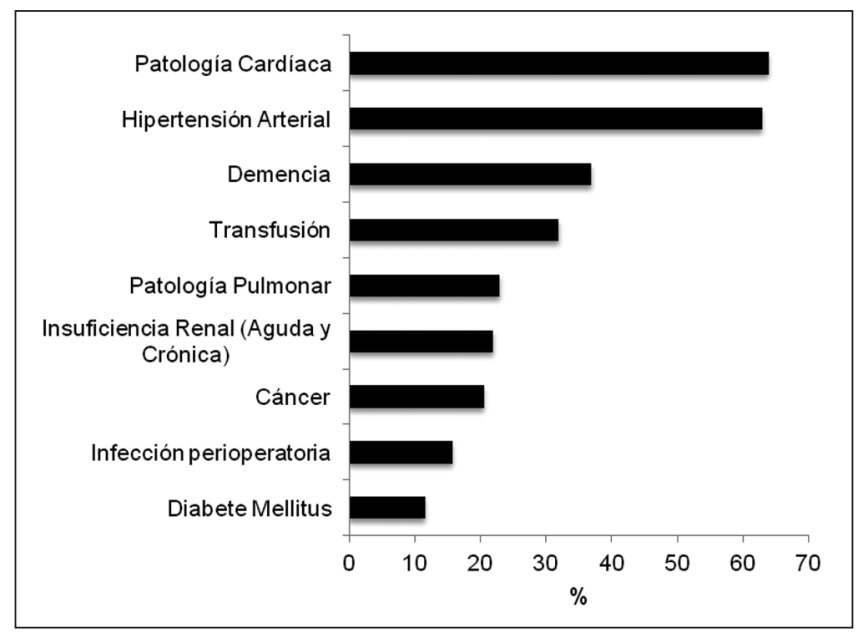

Figura 1. Prevalencia (\%) de patologías agregadas en pacientes nonagenarios.

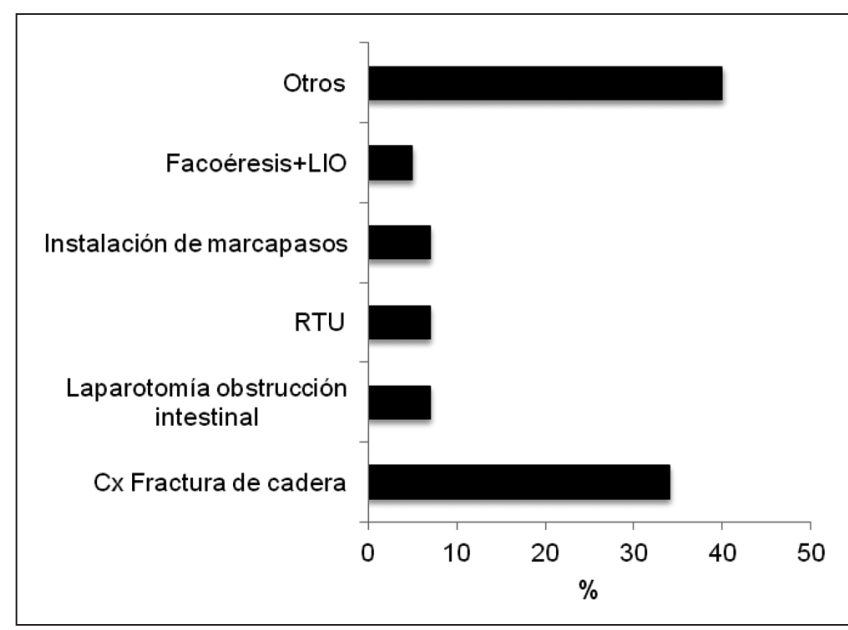

Figura 2. Procedimientos en pacientes nonagenarios (\%).

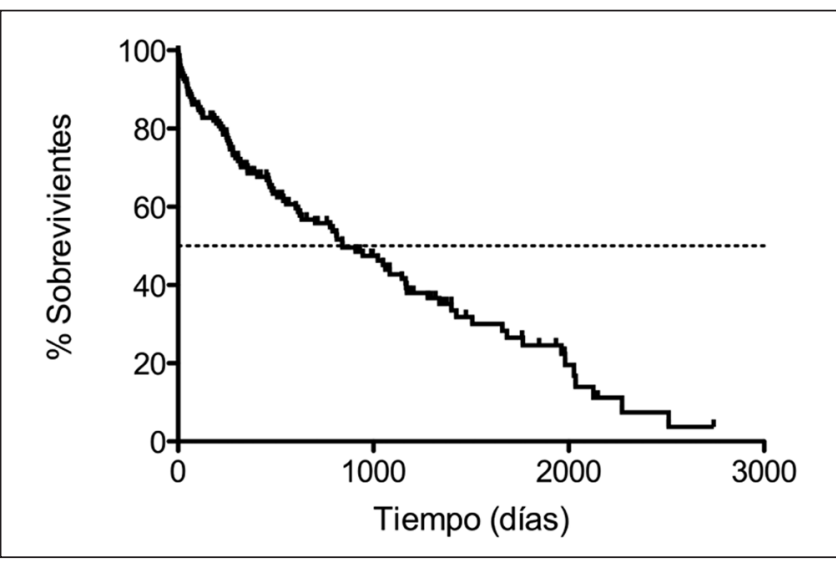

Figura 3. Curva de sobrevida de nonagenarios quirúrgicos. pacientes nonagenarios, según método de Kaplan-Meier5.

Se define como función de riesgo a la tasa instantánea de muerte en un punto dado del tiempo. La función de sobrevida postoperatoria de los pacientes nonagenarios mostró un ajuste adecuado a una distribución de Gompertz ${ }^{6}$, lo que matemáticamente se demuestra por una relación lineal entre el logaritmo natural de la función de riesgo y el tiempo ${ }^{6}$ (Figura 4).

Si se realiza una regresión lineal del logaritmo natural de la función de riesgo con el tiempo como variable explicativa se obtiene un $\mathrm{R}^{2}$ de 0,9659 , lo que demuestra la existencia de una relación lineal entre ellos y, por tanto, un buen ajuste de los datos a una distribución de Gompertz.

Lo anterior permitió realizar regresiones paramétricas con las variables registradas y así identificar los factores pronósticos significativos. Al realizar análisis univariado resultaron ser factores de mal pronóstico la presencia de Clase III de la clasificación ASA, patología cardiaca, pulmonar, alteración de conciencia e infección post-operatoria. Los Hazard Ratios de estos factores pronósticos evidencian que existe el doble de mortalidad en un mismo período de tiempo en los pacientes aquejados de dichas patologías. No hubo diferencia entre la sobrevida de hombres y mujeres, ni entre anestesia regional y general en relación a anestesia local (Tabla 2).

Se realizó análisis multivariado y a través del método step-wise fueron seleccionadas las variables que en forma conjunta resultaran de valor pronóstico. De esta manera resultaron significativas la presencia de transfusión, cáncer, ASA III, patología cardiaca y alteración de conciencia (Tabla 3 ).

Aunque no fue parte de los objetivos de este estudio, debido al incremento progresivo en el número de cirugías en la Clínica Dávila es importante especificar que durante el período estudiado (años 2001-2010), las tasas anuales de anestesia 


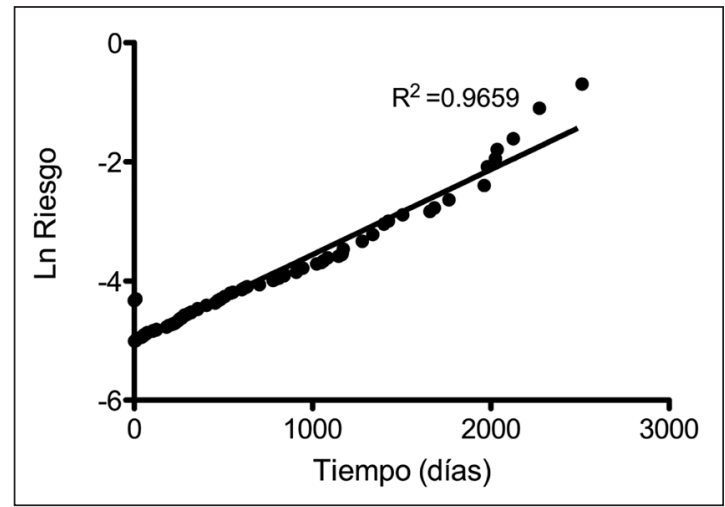

Figura 4. Logaritmo natural del riesgo versus tiempo.

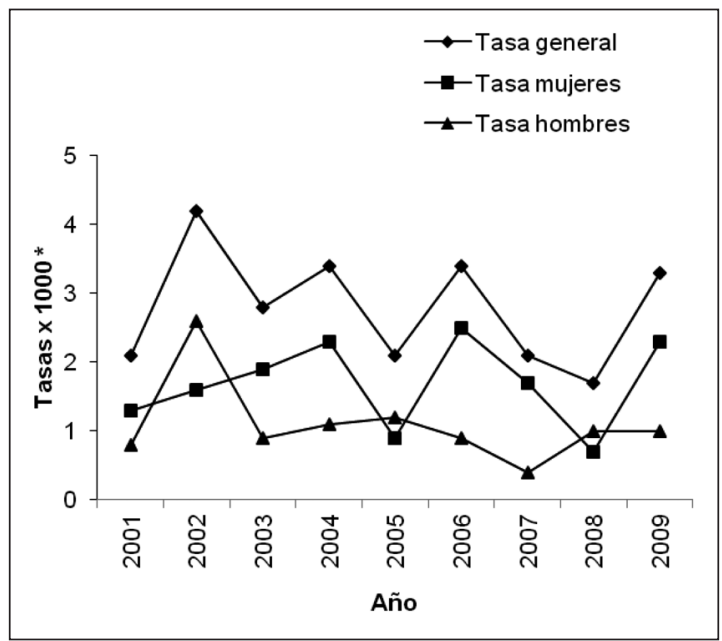

Figura 5. Anestesia en nonagenarios (*tasas x 1.000 anestesias en adultos/año).

en pacientes mayores de 89 años se mantuvieron estables entre 2 y 4 por 1.000 anestesias en adultos (Figura 5). Para analizar la tendencia de dichas tasas en el tiempo se recurrió a regresión de Poisson, lo mismo sucedió al analizarlas según sexo (Tabla 4).

\section{Discusión}

En teoría no se espera que un paciente de noventa años de edad y más tenga una sobrevida muy prolongada, sin embargo, los pacientes de este grupo etáreo que se operan son un grupo especial de nonagenarios, habitualmente los más sanos y por ende con mayor probabilidad de so-
Tabla 2. Análisis univariado

\begin{tabular}{|lcc|}
\hline Factor pronóstico & $\begin{array}{c}\text { Hazard Ratio } \\
\text { [IC 95\%] }\end{array}$ & $\begin{array}{c}\text { Valor } \\
\text { de p }\end{array}$ \\
\hline Edad & $0,98[0,90 ; 1,07]$ & 0,743 \\
\hline Sexo masculino & $1,35[0,89 ; 2,06]$ & 0,157 \\
\hline ASA II & $1,03[0,48 ; 2,20]$ & 0,933 \\
\hline ASA III & $2,24[1,03 ; 4,86]$ & $0,041^{*}$ \\
\hline ASA IV & $1,43[0,49 ; 4,12]$ & 0,508 \\
\hline Anestesia regional & $1,10[0,66 ; 1,82]$ & 0,709 \\
\hline Anestesia general & $1,29[0,72 ; 2,32]$ & 0,384 \\
\hline Cirugía de urgencia & $0,85[0,56 ; 1,29]$ & 0,463 \\
\hline Transfusión & $1,62[1,04 ; 2,52]$ & $0,033^{*}$ \\
\hline Patología cardiaca & $1,99[1,23 ; 3,21]$ & $0,005^{*}$ \\
\hline Patología pulmonar & $1,95[1,22 ; 3,13]$ & $0,006^{*}$ \\
\hline Patología renal & $1,37[0,84 ; 2,26]$ & 0,200 \\
\hline Diabetes & $1,05[0,56 ; 1,98]$ & 0,879 \\
\hline Alteración de conciencia & $2,11[1,38 ; 3,22]$ & $0,001^{*}$ \\
\hline Hipertensión arterial & $1,13[0,73 ; 1,76]$ & 0,571 \\
\hline Cáncer & $1,45[0,89 ; 2,38]$ & 0,134 \\
\hline Infección perioperatoria & $2,21[1,33 ; 3,66]$ & $0,002^{*}$ \\
\hline
\end{tabular}

*valor $p$ estadísticamente significativo. Variables Dummy.

Tabla 3. Análisis multivariado*

\begin{tabular}{|lcl|}
\hline Factor pronóstico & $\begin{array}{c}\text { Hazard Ratio } \\
\text { [IC 95\%] }\end{array}$ & $\begin{array}{c}\text { Valor } \\
\text { de p }\end{array}$ \\
\hline Transfusión & $1,79[1,13 ; 2.83]$ & 0,014 \\
\hline Cáncer & $2,70[1,55 ; 4.70]$ & 0,000 \\
\hline ASA III & $1,95[1,21 ; 3.15]$ & 0,006 \\
\hline Patología cardiaca & $1,91[1,16 ; 3.16]$ & 0,012 \\
\hline Alteración de conciencia & $2,10[1,32 ; 3.22]$ & 0,001 \\
\hline
\end{tabular}

*Selección de variables a través del método step-wise.

Tabla 4. Tendencias en tasas de anestesia en nonagenarios (años 2001-2010)

\begin{tabular}{|lccc|}
\hline Tasa & Tendencia & [IC 95\%] & p - value \\
\hline Tasa general & $-2 \%$ & {$[-7 ;+4 \%]$} & 0,703 \\
\hline Tasa mujeres & $+3 \%$ & {$[-5 ;+10 \%]$} & 0,541 \\
\hline Tasa hombres & $-8 \%$ & {$[-16 ;+0,1 \%]$} & 0,095 \\
\hline
\end{tabular}


brevivir, de manera que al compararlos con sus pares no quirúrgicos se incurriría en un sesgo de selección tipo sesgo de Berkson ${ }^{7,8}$, que consiste en una asociación diferencial entre la exposición y el outcome en los grupos control y en estudio. Por otro lado, no se dispone de datos de sobrevida de la población general nonagenaria en Chile. Un estudio reciente ${ }^{9}$ mostró una mortalidad de $75,5 \%$ a 5 años en una cohorte de nonagenarios residentes en un municipio de Barcelona, lo cual es concordante con nuestros resultados.

Nuestra institución atiende a un segmento socioeconómico de la población intermedio entre la población hospitalaria pública (MINSAL) y de las clínicas privadas. Queda abierta la interrogante respecto a la sobrevida de pacientes nonagenarios quirúrgicos, ajustando por nivel socioeconómico.

La proporción de mujeres en la cohorte fue mayor que la de hombres, pero no hubo diferencia según sexo en la sobrevida, lo cual fue notorio puesto que el riesgo de muerte en todo grupo etáreo es siempre mayor en los hombres ${ }^{10}$.

También destacó que los pacientes clase IV de la clasificación ASA no presentaran menor sobrevida, puesto que ésta es una condición ominosa, de mayor gravedad médica. Van de Kerhove ${ }^{11}$, en un estudio retrospectivo, tampoco encontró diferencias en la sobrevida según la clasificación de ASA en una cohorte de 155 nonagenarios sometidos a cirugía de fractura de cadera.

Es aceptado en demografía de poblaciones envejecidas que el modelo probabilístico que mejor describe la mortalidad es la distribución de Gompertz $^{12,13}$; esto implica una disminución exponencial de la sobrevida. La sobrevida de nuestros pacientes se ajustó bastante precisamente a dicha distribución de sobrevida.

Nuestros resultados están muy influenciados por el tipo de cirugía que predominó en la muestra: fractura de cadera. Warner ${ }^{14}$ encontró en 224 nonagenarios sometidos a cirugía que las patologías más frecuentes eran las digestivas y la fractura de cadera ( $22 \%$ cada una). Söderqvist ${ }^{15}$ encontró en una cohorte prospectiva exclusivamente compuesta de 1.944 pacientes con fractura de cadera (pero que incluía pacientes mayores de 66 años) 38\% de mortalidad a 2 años. Fue destacable también que el tipo de anestesia (general, regional o local) no tuviera efecto en la sobrevida de los pacientes. Habitualmente se prefiere el uso de anestesia regional (cuando es posible) en los paciente añosos, pese a que no se ha demostrado un efecto beneficioso en la función cognitiva postoperatoria al comparar anestesia regional versus anestesia general ${ }^{16}$. En nuestro estudio el 50\% de los pacientes recibió anestesia regional, puesto que uno de los procedimientos más frecuentes fue cirugía de fractura de cadera. El predominio de la cirugía de urgencia (60\%) sobre la electiva también puede atribuirse a la alta frecuencia de cirugía de fractura de cadera; en nuestra institución esta patología se considera una urgencia por las consecuencias potenciales que tiene retardar la cirugía ${ }^{17,18}$.

Fue notoria la alta proporción de patología cardiaca (insuficiencia cardiaca, arritmias y cardiopatía coronaria) en este grupo de pacientes. Esto no es extraño, puesto que el riesgo de padecer insuficiencia cardiaca aumenta con la edad ${ }^{19}$ y según un reporte reciente ${ }^{20}$, la enfermedad cardiovascular sigue siendo la primera causa de mortalidad en pacientes mayores de 65 años en Estados Unidos de Norteamérica.

La prevalencia de deterioro cognitivo en este estudio fue de $37 \%$. Marín ${ }^{21}$ en un estudio nacional encontró $77 \%$ de trastornos en la evaluación mental en mujeres nonagenarias institucionalizadas, lo que sugiere que los nonagenarios quirúrgicos eran más saludables. La alteración de conciencia también ha sido asociada por otros autores con una menor sobrevida; sin embargo, Dolan ${ }^{22}$ no encontró que el delirio en la admisión fuera un predictor significativo de mortalidad después de ajustar por otros confusores. Lee ${ }^{23}$, por otra parte, detectó menor sobrevida a 40 meses post cirugía de cadera en pacientes que presentaron delirio. Robinson $^{24}$ también encontró mayor mortalidad después de una cirugía mayor en pacientes que habían presentado delirio.

La presencia de transfusión se asoció en forma independiente y multivariada con un mayor riesgo de muerte; ésta pudo ser reflejo de una cirugía más cruenta y complicada o de una patología basal como anemia o coagulopatía. En una población de pacientes ancianos con alta prevalencia de patología cardiovascular, sometidos a una cirugía, es muy probable que no se restrinjan las transfusiones, con el fin de evitar la isquemia miocárdica. Carson ${ }^{25}$, en un estudio de 1.958 pacientes que rehusaron ser transfundidos, detectó que bajo un umbral de hemoglobina preoperatoria de $10 \mathrm{~g} / \mathrm{dl}$ había mayor mortalidad en el grupo de pacientes con enferme- 
dad cardiovascular. El mismo autor ${ }^{26}$ demostró que no existía diferencia en la mortalidad en una población de pacientes con un promedio de edad de 81,6 años y $62,9 \%$ de prevalencia de patología cardiovascular sometidos a una estrategia liberal o restringida de transfusión. Ezekowitz ${ }^{27}$ demostró que en pacientes portadores de insuficiencia cardiaca la anemia era un factor de mal pronóstico.

Se observó una prevalencia de cáncer de $20 \%$ en los nonagenarios quirúrgicos. Este hallazgo no sería extraño, puesto que según las estadísticas de la American Cancer Society, la incidencia de cáncer incrementa con la edad, alcanzando un máximo entre los 65-74 años ${ }^{28}$. El cáncer en forma independiente no fue factor pronóstico significativo, pero si lo fue en el análisis multivariado.

Una falencia de este estudio fue no haber investigado el estado nutricional de los pacientes. Faxen-Irving ${ }^{29}$ encontró que un IMC menor que $23 \mathrm{~kg} / \mathrm{m}^{2}$ estaba asociado con un aumento de la mortalidad en forma independiente de otras comorbilidades, en un grupo de pacientes de 80 \pm 7 años de edad. Si consideramos que nuestros pacientes eran quirúrgicos (que requerían cicatrización) pudiera elucubrarse que el estado nutricional hubiera sido una variable de importancia capital en la sobrevida; además, la desnutrición es muy prevalente en los pacientes ancianos ${ }^{30} \mathrm{y}$ debiera considerarse en futuros estudios sobre el tema.

En conclusión, la cohorte de pacientes nonagenarios operados en la Clínica Dávila en el período 2001-2010 presentó 50\% de mortalidad a 2 años de la cirugía. Fueron factores de mal pronóstico la presencia de un cáncer concomitante, patología cardiaca, trastornos de conciencia, haber sido transfundido y pertenecer a la clase III de la clasificación de estado físico de la Sociedad Americana de Anestesiología.

\section{Referencias}

1. Instituto Nacional de Estadísticas. Censo de Población y Vivienda, Chile 2002.

2. Hosking M P, Warner M A, Lobdell Creig M, Offord K P, Melton J. Outcomes of surgery in patients 90 years old and older. JAMA 1989; 261: 1909-15.

3. Djokovic J L, Hedley-Whyte J. Prediction of outcomes of surgery and anesthesia in patients over 80. JAMA 1979; 242: 2301-6.

4. American Society of Anesthesiologists. New classification of physical status. Anesthesiology 1963; 24: 111.
5. Lee ET, Wenyu Wang J. Statistical methods for survival data analysis, Third Edition. Hoboken, NJ. Wiley series in probability and statistics. 2003.

6. Cavada G. Algunas distribuciones de probabilidad usadas en el análisis de sobrevida. En Gabriel Cavada Chacón. Análisis de Sobrevida. División de Bioestadística y Demografía. Escuela de Salud Pública, Universidad de Chile; 2007. p 10-20.

7. Delgado-Rodríguez M, Llorca J. Bias (Glossary). J Epidemiol Community Health 2004; 58: 635-41.

8. Wacholder S, Silverman DT, McLaughlin, Mandel JS. Selection of Controls in Case-Control Studies II. Types of Controls. Am J Epidemiol 1992; 135: 1029-41.

9. Formiga F, Ferrer A, Chivite D, Rubio-Rivas M, Cuerpo S, Pujol R. Predictors of long-term survival in nonagenarians: The NonaSantfeliu study. Age ageing 2011; 40: 111-6.

10. Woloshin S, Schwartz LM, Welch G. The risk of death by age, sex and smoking status in the United States: Putting health risks in context. J Natl Cancer Inst 2008; 100: 84553.

11. Van der Kerkhove MP, Antheunis PS, Luitse JSK, Goslings JC. Hip fractures in nonagenarians: Perioperative mortality and survival. Int J Care Injured 2008; 39: 2448.

12. Comfort A. Aging. The biology of senescence Routledge Kegan Paul, London. 1964.

13. Finch CE. Longetivity, Senescence and the genome Univ of Chicago Press, Chicago. 1990.

14. Warner MA, Hosking MP, Lobdell CM, Offord KP, Melton LJ. Surgical procedures among those $\geq 90$ years of age. Ann Surg 1988: 207: 380-6.

15. Söderqvist A, Ekström W, Ponzer S, Pettersson H, Cederhom T, Dalén N, Hedström M, Tidermark J. Prediction of mortality in elderly patients with hip fractures: a two year prospective study of 1.944 patients. Gerontology 2009; 55: 496-504.

16. Williams-Russo P, Sharrock NE, Matis S, Szatrowski TP, Charlson ME. Cognitive effects after epidural vs general anesthesia in older adults. A randomized trial. JAMA 1995; 274: 44-50.

17. Gillespie WJ. Extracts from "Clinical evidence": Hip fracture. BMJ 2001; 322: 968-75.

18. Novack V, Jotkowitz V, Etzion O, Porath A. Does delay in surgery after hip fracture leads to worse outcomes? A multicenter Survey. Int J Quality in Health Care 2007; 19: 170-6.

19. Kannel B. Incidence and epidemiology of heart failure. Heart Fail Rev 2005; 5: 167-73.

20. Murphy SL, Xu J, Kochanek KD. National Vital Statistical Reports 2012; 60 (4). 
Sobrevida postoperatoria en nonagenarios - D. Ojeda et al

21. Marín PP, Gac H, Hoyl T, Carrasco M, Duery P, Cabezas $\mathrm{M}$, et al. Estudio comparativo de mujeres mayores de noventa años y ancianas menores institucionalizadas. Rev Med Chile 2004; 132: 33-9.

22. Dolan MM, Hawkes WG, Zimmerman SI, Morrison RS, Gruber-Baldini AS, Hebel JR, et al. Delirium on hospital admission in aged hip fractured patients: prediction of mortality and 2-years functional outcomes. J Gerontol A Sci Biol Med Sci 2000; 55: M 527-34.

23. Lee KH, Ha YC, Lee YK, Kang H, Koo KH. Frequency, risks factors and prognosis of prolonged delirium in elderly patients after hip fracture surgery. Clin Ortho Relat Res 2011; 469: 2612-20.

24. Robinson TN, Raebum CD, Tran ZV, Angles EM, Brenner LA, Moss M. Postoperative delirium in the elderly: Risks factors and outcomes. Ann Surg 2009; 249: 173-8.

25. Carson JL, Duff A, Poses RM, Berlin JA, Spencer RK, Trout R, et al. Effect of anaemia and cardiovascular disease in surgical mortality and morbidity. Lancet 1996; 348: 1055-60.
26. Carson JL, Terrin ML, Noveck H, Sanders DW, Chaitman BR, Rhoads GG, et al. Liberal or restrictive transfusion in high-risk patients after hip surgery. N Engl J Med 2011, 365: 2453-62.

27. Ezekowitz JA, McAlister FA, Armstrong PW. Anemia is common in heart failure and is associated with poor outcomes: insights from a cohort of 12065 patients with new-onset heart failure. Circulation 2003; 107: 223-5.

28. Ries LAG, Eisner MP, Kosary CL, Hankey BF, Miller BA, Clegg L, et al (Eds). SEER Cancer statistics review 1975_2000, National Cancer Institute. Bethesda, MD, http://seer.can.gov/csr/1975_2000/2003.

29. Faxen-Irving G, Basun H, Cederholm T. Nutritional and cognitive relationship and long-term mortality in patients with various dementia disorders. Age Ageing 2005; 34: 136-41.

30. Kagansky N, Berner Y, Koren-Morag N, Perelman L, Knobler H, Levy S. Poor nutritional habits are predictors of poor outcome in very old hospitalized patients. Am J Nutr 2005; 82: 784-91. 